\title{
Targeting tuberculosis using structure-guided fragment-based drug design
}

Vitor Mendes and Tom L. Blundell ${ }^{*}$

Department of Biochemistry, University of Cambridge, Cambridge CB21GA, UK

*Corresponding author: Blundell, T.L. (tom@cryst.bioc.cam.ac.uk).

Teaser: Fragment-based drug discovery has produced a number of potent inhibitors against multiple tuberculosis targets. Here, we review the successes, opportunities and challenges of this approach in tuberculosis drug discovery.

Keywords: Mycobacterium; tuberculosis; fragments; FBDD; drug discovery.

Compounds discussed in this article: Benzofuran-2carboxylic acid; 5-methoxy-3Hindole; 2-(5-methoxy-2-(((4-(trifluoromethyl)benzyl)sulfonyl)carbamoyl)-1H-inden-1yl)acetic acid; 3-cyclopentyl-1-(pyrrolidin-1-yl)propan-1-one; $\quad N$-methyl-1-(4(piperidin-1-yl)phenyl)methanamine; 4-(4-(3-oxo-3-(pyrrolidin-1-yl)propyl)piperidin-1yl)benzonitrile; 4-(methylthio)-2-oxo-6-(thiophen-2-yl)-1,2-dihydropyridine-3carbonitrile; 6-(3-((4-isopropylbenzyl)oxy)phenyl)-4-(methylsulfonyl)-2-oxo-1,2dihydropyridine-3-carbonitrile; 4-(1H-1,2,4-triazol-1-yl)phenol; $\quad 4$-(5-amino-4-(3'amino-[1,1'-biphenyl]-3-yl)-1 $H$-pyrazol-3-yl)phenol. 
Fragment-based drug discovery is now widely used in academia and industry to obtain small molecule inhibitors for a given target and is established for many fields of research including antimicrobials and oncology. Many molecules derived from fragment-based approaches are already in clinical trials and two - vemurafenib and venetoclax - are on the market, but the approach has been used sparsely in the tuberculosis field. Here, we describe the progress of our group and others, and examine the most recent successes and challenges in developing compounds with antimycobacterial activity. 


\section{Introduction}

There is an urgent need to find novel therapies to fight the global epidemic of tuberculosis (TB). The rise of multidrug-resistant (MDR)-TB and extensive-drugresistant (XDR)-TB strains, exacerbated by a deadly synergy with HIV, exerts a heavy toll in the developing world. Current TB therapy relies on a combination of drugs (isoniazid, rifampicin, pyrazinamide and ethambutol) that were discovered 50-60 years ago. Although up to $95 \%$ of patients can be cured with this therapy, the drugs must be taken for a period of 6 months for drug-sensitive TB. Length of treatment and mortality rate increase substantially for MDR-TB and XDR-TB strains [1]. Several highthroughput target-based and phenotypic screening campaigns were performed during the past decade. Although target-based campaigns identified a number of leads that show high potency in vitro, most did not show any translation to an in vivo effect and in the phenotypic screens the hit rate has been lower than expected. This has been attributed to the limited chemical diversity within those libraries and also to the selection of molecules that are drug like, with smaller and larger molecules often omitted [2,3]. Furthermore, there is a substantial rate of attrition of new drugs during clinical trials because of the complex nature of TB. Multiple replication states of Mycobacterium tuberculosis, together with a diverse set of lesions with different local environments, can be present within a patient, leading to problems of penetration being insufficient to achieve efficacious concentrations [4,5]. Therefore, there is a need to find and develop new drugs to fight TB using different approaches that allow us to explore more chemical space.

Fragment-based drug discovery (FBDD) is a powerful and now widely used approach to create novel high-quality drug-like molecules [6-9]. This approach relies on screening a library consisting of small molecules (150-300 Da) against a target protein, using a 
variety of biochemical, biophysical and structural biology methods. The low molecular weight of fragments represents a decrease of complexity and allows an efficient exploration of chemical space even when using small libraries of 1000 fragments. Although the fragments usually bind weakly, they tend to bind to hotspots forming well-defined interactions with the target protein [10]. Thus, fragments can be subsequently elaborated into larger molecules with high affinity [10].

\section{FBDD approach}

Our approach to fragment screening involves two initial stages: first a screen to identify hits and then determination of the 3D structures of protein-fragment complexes, followed by a study of thermodynamics and kinetics of the binding process $[6,7,9,10]$ (Figure 1). Owing to the low affinity shown by most fragments, high concentrations have to be used in the screening process. Nevertheless, fragments present in the libraries are selected for their aqueous solubility and therefore the high concentrations used generally do not lead to assay problems. This approach has been described in depth before in the references cited above and readers should consult these for more information. We, therefore, will only give a brief overview while focusing on updating the information to current standards.

For the initial identification of hits, biophysical methods are employed for screening fragments. In our laboratory a popular approach is differential scanning fluorimetry (DSF), often known as thermal shift, a technique that allows the detection of compounds that increase the melting temperature (unfolding temperature) of a target protein on binding by promoting protein stability [11]. Pre-screening the target protein with natural ligands and a diverse set of buffers, $\mathrm{pH}$ range and additives, such as salts, 
glycerol and polyethylene glycol (PEG), is indispensable, not only to assess the suitability of the technique for the target protein but also to find the right conditions for the screen. DSF can be adapted to rank compounds by testing the hits at multiple concentrations and used to calculate an approximate binding affinity [12].

DSF hits are usually confirmed by ligand-based 1D ${ }^{1} \mathrm{H}$ NMR spectroscopy Carr-PurcellMeiboom-Gill (CPMG), saturation transfer difference (STD) and water ligand observed gradient spectroscopy (WaterLOGSY) [13-16]. However, NMR spectroscopy can also be used to perform initial screening, possibly using cocktails of two or more fragments to accelerate the procedure. Other complementary screening and ranking techniques can be used depending on the project characteristics. Surface plasmon resonance (SPR) is useful for direct screening of fragment libraries especially when obtaining protein in large amounts is a problem [17]. Competition assays can be performed if a known ligand is bound to the surface of the chip instead and the target protein is then allowed to form a complex with it. Running the fragment solution over the chip in this scenario will free the protein from the chip if the fragments compete with the used ligand.

Biolayer interferometry (BLI) is another method that can be used to screen and rank fragments; it is especially useful for targeting protein-protein interactions [18]. Other techniques such as nanoelectrospray ionization mass spectrometry (ES-MS) [19] and microscale thermophoresis (MST) [20] have also been recently applied to fragment screening and ranking, with both methods correlating well with X-ray crystallography [20,21]. Functional biochemical assays can also sometimes be used as a highthroughput method to screen for inhibitors. This technique is useful for detecting weakly inhibiting fragments by optimizing the assay to work with a high concentration of compounds. 
Once hits have been identified, the 3D structures of the fragment-protein complexes need to be defined. Although X-ray crystallography has been used as a screening technique, usually by soaking cocktails of up to five fragments, it is employed more often to characterize the mode of binding of pre-established hits obtained with the previously described methods. This involves establishing a robust crystallization system that yields highly diffracting crystals (to better than $2.5 \AA$ resolution) with the binding site of the protein exposed in the crystal lattice and soaking those crystals with a high concentration of fragments $(10-50 \mathrm{mM})$. For proteins that suffer large conformational changes upon ligand binding, co-crystallization of protein-fragment complexes can be employed instead or the conformation stabilized by a specific antibody [22].

Although not used as an initial screening technique owing to the large quantities of protein required, isothermal titration calorimetry (ITC) is an invaluable tool to characterize fragment-binding affinities and to characterize the thermodynamics of binding [23]. However, obtaining good titration data from fragments can be challenging because of their weak affinity and this technique is often relegated to later stages in the project when strong binders are available.

In silico methods are widely available and are heavily used in drug discovery, not only in HTS screening campaigns but also as a means to produce structural information about binding modes when X-ray crystal structures of protein-inhibitor complexes are not available. Our group has developed methods that can enhance and complement the current in silico methods with particular application to fragment-based drug discovery. As mentioned before, owing to their small size, fragments interact weakly with the target protein, usually between $0.1-5.0 \mathrm{mM}$, but tend to bind to hotspots that make large contributions to binding affinity [24]. Hotspots usually have the capacity to bind not only one but a variety of fragment-sized molecules [24]. As fragments are elaborated 
into higher affinity compounds, analyzing the target protein hotspots can provide crucial insights into how to improve compounds [24].

Several methods have been described in the literature to map protein hotspots. Our group recently developed a method that identifies the hotspot by sampling atomic hotspots with simple molecular probes to produce fragment hotspot maps [25]. It specifically highlights fragment-binding sites and their corresponding pharmacophores, providing an intuitive visual guide within the binding site. This method can also help generate constraints for docking experiments by ensuring the right interactions are made.

Elaboration of fragment hits for a particular target protein will exploit one or more of the techniques mentioned above. The objective is to establish a continuous cycle of fragment-merging/linking/growing strategies, while maintaining high ligand efficiency (LE) - a measurement of the binding energy per atom of a ligand to its binding partner, such as a receptor or enzyme, typical of fragments. The elaboration of compounds is supported by structural information from X-ray crystal structures and in silico methods, together with characterization of binding by biophysical and functional biochemical assays.

\section{Co-enzyme A synthesis as a TB target}

Co-enzyme $\mathrm{A}$ is an essential and ubiquitous cofactor, key to a large number of metabolic reactions including fatty acid metabolism and the tricarboxylic acid cycle [26]. Predictably, some of the enzymes leading to its synthesis have been found to be 
essential in $M$. tuberculosis and, although these enzymes are also present in humans, sequence identity is low and therefore they are considered good drug targets.

Using the fragment-based approach described above, our laboratory in collaboration with that of Chris Abell has targeted the enzymes PanC, CoaD and CoaBC of the coenzyme A pathway using FBDD, obtaining several fragment hits by DSF that have been confirmed by X-ray crystallography and ITC (Figure 2) [27,28]. PanC is an enzyme that synthesizes pantothenate (vitamin $\mathrm{B}_{5}$ ), the precursor for co-enzyme A synthesis. Initial fragment hits for this enzyme were found to bind to two different subsites of the active site, with one fragment occupying the pantoate site and a phosphate site of ATP, and the other binding to the adenine site of ATP (Figures 2a and 2b, respectively). As shown in Figure $2 \mathrm{c}, \mathrm{d}$, the fragment hits are bound to hotspot regions of PanC predicted by our computational method [25]. To explore better the possibilities given by the hits, fragment-linking and fragment-growing strategies have been employed, resulting in low micromolar affinity compounds (Figure 2e-g) [28]. As has been the case in other fragment-to-lead campaigns [10], both strategies allowed the development of inhibitors with high LE [28]. Further improvements in compound affinity were guided by X-ray crystal structures with the final compounds reaching nanomolar affinity [29] (Figure 2h). Although the final compounds are potent in vitro and gene validated as essential, the results in cell-based assays do not relate to the potency of the compounds (unpublished data).

\section{Hitting cell wall synthesis}

Mycobacteria possess a lipid-rich cell wall composed of a covalently linked peptidoglycan-arabinogalactan-mycolic acid complex, the latter being a major contributor to the permeability, fluidity and pathogenicity of mycobacteria [30]. The 
synthesis of this complex cellular envelope is well understood with many essential steps, some of them already medically explored by drugs such as isoniazid (mycolic acid synthesis), ethambutol (arabinogalactan synthesis), ethionamide (mycolic acid synthesis) and D-cycloserine (peptidoglycan). Owing to the essentiality of the pathways and to their already-proven therapeutic potential, it is not surprising that many new drug discovery campaigns have targeted them. Furthermore, the fact that some biosynthetic steps of these pathways are located outside the cell membrane makes them more attractive because they are more accessible to drugs than intracellular targets.

Fragment-based approaches have recently been used to target the pathways leading to mycolic acid synthesis, focusing not only on fatty acid synthase II (FAS II) components but also on the periplasmic stages of the process, more specifically the antigen 85 (Ag85) complex. This periplasmic complex, composed of three different but partially redundant enzymes, is responsible for the attachment of trehalose monomycolate (TMM) to either arabinogalactan to form cell-wall-bound mycolates or to another TMM molecule thus forming trehalose dimycolate (TDM), also known as cord factor $[31,32]$. Mutation studies in M. tuberculosis showed that, although the enzymes are partially redundant, a mutant lacking Ag85C showed a 40\% decrease in cell-wall-linked mycolic acids and altered cell wall permeability. It has not been possible to generate double knockout mutants, pointing to synthetic lethality of the mutations [33,34]. Furthermore, because the three enzymes are very similar ( $70 \%$ sequence identity), it is possible that a single compound will be able to hit more than one of the Ag85 components at the same time. A fragment-based approach was used to identify high ligand efficiency substrate-site binders of $\mathrm{Ag} 85 \mathrm{C}$ that inhibited the growth of drug-sensitive $\mathrm{TB}$ and MDR-TB [35]. The approach used by Scheich and colleagues was based on ${ }^{15} \mathrm{~N}-\mathrm{HSQC}$ NMR spectroscopy as a tool to screen a diverse fragment library of 5000 compounds, 
followed by antibacterial growth assays of the hits against mycobacterial strains and control strains to account for off-target inhibition and finally fragment elaboration [35]. The authors initially identified six fragment hits by NMR screening; one of these fragment hits (Figure 3a) showed growth inhibition for Mycobacterium smegmatis with an MIC of 50-100 $\mu \mathrm{g} / \mathrm{ml}$. However, testing of this compound against M. tuberculosis H37Rv and an MDR strain showed an MIC of $500 \mu \mathrm{g} / \mathrm{ml}$. Although the authors could not obtain crystal structures of Ag85C-inhibitor complexes to guide fragment elaboration, a systematic approach was used by successively testing different chemical substitutions in similar analogs (Figure $3 b-d$ ) to increase lead potency. The best compound (Figure 3d) showed an MIC of $20-50 \mu \mathrm{g} / \mathrm{ml}$ against $M$. tuberculosis H37Rv and an MDR strain [35]. Although limited, the chemical modifications explored by the authors provided a tenfold improvement of the MIC and revealed inhibitors with high LE and low molecular weight that have the potential to provide a basis for further elaboration.

KasA is an essential component of the FAS II system involved in mycolic acid synthesis [36]. Attention was given to this target after the isolation of natural products that inhibit Kas enzymes [37,38]. Using a fragment-sized inhibitor of KasA (thiolactomycin) (Figure 3e) as a starting point, Kapilashrami et al. employed a fragment-growing strategy relying on information from transient 1D NOE spectroscopy, enzymatic assays and fluorescence-based assays to improve the affinity of the initial compound [39] (Figure 3f-h). Their results showed that an elaboration of the thiolactone ring at $\mathrm{C} 3$ enhanced the potency of the inhibitors. The subsequent modification of the methyl group with larger groups led to an increase of affinity $\left(K_{\mathrm{d}}\right)$ from $\sim 220 \mu \mathrm{M}$ to $\sim 10 \mu \mathrm{M}$ [39]. However, LE decreased substantially from that of the initial compound to the most potent (Figure 3e,h). Nevertheless, one of their 
intermediate compounds still retained high LE with $\sim 22 \mu \mathrm{M} K_{\mathrm{d}}$ (Figure $3 \mathrm{~g}$ ). It is possible that further exploration of chemical modifications in that position could increase further the affinity while retaining high LE.

\section{New drugs for old targets}

The enoyl-acyl carrier protein reductase (InhA), a component of the FAS II complex involved in mycolic acid synthesis, is the target for a first-line TB therapeutic, isoniazid, a pro-drug that requires activation by the heme enzyme catalase peroxidase KatG. The activated drug then reacts with NAD to form an isoniazid-NAD adduct that strongly binds to the enzyme [40]. Resistance to isoniazid can be largely explained by defects in the activating enzyme and, less commonly, in InhA itself or in the upstream promoter region of InhA [41]. Therefore it makes sense to explore inhibition of InhA through other means, by directly hitting the substrate site, an approach that has been tested by others [42-44]. We have explored this idea using our fragment-based approach (unpublished data). Our fragment library was screened against InhA in the presence of NAD using DSF and ligand-based NMR (CPMG, STD and WaterLOGSY). Binding modes of fragment hits were confirmed by X-ray crystal structure analysis (Figure 3i,j). Fragments were ranked by an enzymatic assay, and then elaborated successively to improve inhibition. The diverse chemical properties of the fragments allowed us to explore new chemical scaffolds and find novel approaches to inhibit InhA.

\section{Teaching new tricks to old drugs: boosting the ethionamide effect by hitting EthR}

Ethionamide is a second-line drug currently used to treat TB that also hits InhA. Like isoniazid it is a pro-drug that requires activation by a mono-oxygenase EthA and that subsequently will react with NAD to form a high affinity adduct [45]. EthA expression levels are therefore of utmost importance for activation of ethionamide. The high level 
ethionamide dosage used in current MDR-TB treatments is known to cause liver toxicity issues. Therefore, by increasing EthA expression levels, lower doses of this drug could be used in TB therapy, which would limit toxicity. It is possible to limit repression of EthA by targeting EthR, a TetR family repressor that modulates EthA expression, with high affinity ligands, boosting the ethionamide killing effect, as demonstrated by Willand et al. in mice infected with $M$. tuberculosis [46]. With this in mind, attempts to develop potent EthR binders with distinct fragment approaches were made by our group and by Villemagne and colleagues [47-49]. EthR possesses a long and hydrophobic binding site that accommodates a fatty acid and was considered to offer little prospect for H-bond interactions with ligands [50]. Owing to the efficient chemical space exploration that characterizes fragment-based drug discovery, H-bond opportunities that are not explored by the natural ligand in the hydrophobic cavity were identified in the process of fragment screening [49]. Using a fragment-merging strategy (Figure 4k-n), supported by biophysical methods (DSF, ITC and SPR) and X-ray crystallography, Nikiforov and colleagues achieved a $\sim 100$-fold increase in potency [47]. However, none of the developed compounds synthesized in this work showed an ethionamide-boosting effect against $M$. tuberculosis [47], whereas the initial fragment was shown to have a boosting effect [49]. Other authors were more successful and designed several lead inhibitors for EthR that are able to penetrate the thick mycobacterial cell wall and that show an ethionamide-boosting effect in vitro and in macrophage infection models $[48,49]$. However, fragment-like compounds could prove effective where more than one copy can be bound with high affinity to targets that have multiple hotspots [47-49] (Figure 4e,f).

\section{Other targets}




\section{Dehydroquinolase}

Other pathways and enzymes have been explored in TB drug discovery using fragmentbased approaches. The shikimate pathway is present in microorganisms and plants, where it leads to the synthesis of key aromatic compounds including aromatic amino acids. This metabolic route has received some attention in TB drug discovery, however a fragment approach was used only for dehydroquinolase, the third enzyme of the pathway that catalyzes the reversible dehydration of 3-dehydroquinate to 3dehydroshikimate. Fragment-sized compounds, previously identified in a virtual screening campaign [51], were used by Tran et al. as their starting library and they further selected them by in silico molecular docking, choosing those that mimicked the anhydroquinate core but that had higher hydrophobicity [52]. Subsequent fragment elaboration guided by in silico molecular docking led to the identification of several inhibitors with activity in cell-based assays with the best showing a $K_{\mathrm{i}}$ of $\sim 18 \mu \mathrm{M}$ and $\mathrm{MIC}_{50}$ of $\sim 10 \mu \mathrm{M}$ against M. tuberculosis $\mathrm{H} 37 \mathrm{Ra}$ strain [52].

\section{Thymidylate kinase}

Thymidylate kinase (TMK), an enzyme involved in DNA synthesis that produces thymidine 5'-diphosphate from ATP and thymidine 5'-monophosphate, has been found to be essential for $M$. tuberculosis [53]. Target-focused drug discovery campaigns performed on this enzyme have identified some inhibitors but all of the compounds obtained were either thymidine monophosphate analogs or contained a thymidine moiety [53]. Naik and colleagues performed a fragment screen using NMR and a biochemical assay that led to identification of new scaffolds inhibiting TMK [54]. They prioritized a 3-cyanopyridone-containing fragment for chemical elaboration (Figure 4a,b). Although they could not obtain X-ray crystal structures of the fragment, 
molecular docking was used instead to predict the mode of binding. The subsequent development of the fragment, supported by X-ray crystal structures of key compounds, allowed them to obtain nanomolar-potency lead compounds against this enzyme that retained high LE. These compounds differed from the substrate analogs and some proved to be active against $M$. tuberculosis [54] (Figure 4c-f).

\section{Cytochrome P450 121}

M. tuberculosis possesses an unusually high number (i.e., 20) of cytochrome P450 enzymes (CYPs), some of which have been demonstrated to be essential for the survival of the organism [55-57]. CYP121 is an essential CYP, found exclusively in $M$. tuberculosis [57], with known high-affinity binders that show potent activity in vitro and in cell-based assays [58,59]. A significant effort using fragment-based approaches targeting this enzyme led to the identification of several potent and selective inhibitors of CYP121 that have high ligand efficiency [60-63]. A fragment screening campaign was performed by Hudson and colleagues using DSF, NMR and ITC revealing several fragment hits [62]. An example of one of the hits is shown in Figure 4g. This fragment,

which was present in two different binding modes (Figure $4 \mathrm{~g}$ ), was prioritized for a fragment-merging strategy that led to the development of a low micromolar affinity compound but with low LE (Figure 4h) [61]. The low LE led Chris Abell's group to use a retro-fragmentation strategy of compound in Figure $4 \mathrm{~h}$ to assess the group efficiency of its component fragments [60]. This allowed a sequential rebuild of compounds with higher LE. Figure 4i shows one of those compounds that nevertheless had lower affinity than the parent compound. Further elaboration of the compound based on this fragmentation strategy led to the design of a low nanomolar lead that makes polar contacts with the heme iron (Figure 4j) [60]. Despite the high potency of the compounds they did not show antimycobacterial activity over the tested concentration range (1 to 
$100 \mu \mathrm{M})$ and the authors argued that permeability issues and drug-efflux mechanisms might be the cause [60].

\section{Concluding remarks}

M. tuberculosis possesses a complex cell wall that is also a formidable permeability barrier that drugs need to pass. The complex nature of TB in which M. tuberculosis shows multiple replication states within the host adds a further layer of difficulty to TB drug discovery. The TB drug discovery field over the past decade has seen a shift from target-based screens back to whole-cell phenotypic screens in the search for potent inhibitors, owing to the problems faced by hits from target-based approaches in penetrating the cell wall [3]. However, phenotypic screens have not produced a substantial number of hits [2]. Fragment-based approaches have been able to produce potent lead compounds in vitro, some of which show good antimycobacterial activity despite the small number of dedicated studies in TB drug discovery using this methodology $[48,49,54]$. The fragments provide good starting points for rational compound elaboration that explores the protein hotspots. The subsequent structureguided optimization of the fragment hits is able to generate lead compounds that have high ligand efficiency while keeping molecular weight, lipophilicity and other physicochemical properties controlled [54]. This is advantageous for TB drug discovery because cell penetration is a major issue owing to the highly impermeable cell wall, but small hydrophilic compounds have been shown to enter the mycobacteria cell through porins [64]. Elaborating compounds with these properties might lead to drugs that can diffuse through porins and cross the cell wall more easily.

The power of FBDD relies on its ability to screen a vast chemical space using a small library of fragments with good physiochemical properties. This is particularly relevant 
for TB where the complex lifecycle of the pathogen and progression of the disease requires molecules that are often not drug-like. Existing antimicrobials for TB include molecules that are much larger and more lipophilic than those defined as drug-like, as well as others that are much smaller and less lipophilic. Fragment-sized molecules have been used to treat TB for many years. Such examples of this are isoniazid, ethambutol, ethionamide, para-aminosalicilic acid, pyrazinamide, prothionamide, D-cycloserine and thioacetazone. Many of these very small molecules (isoniazid, ethionamide, paraaminosalicylic acid and pyrazinamide) are chemically elaborated once inside the mycobacterium and are inactive against their protein targets without chemical activation. Some authors have therefore argued that reactive fragments should be reincluded in phenotypic screens to take advantage of their capacity to target multiple pathways after activation [65]. The EthR results indicate that activity with fragments could also be achieved with targets offering multiple hotspots and deep pockets providing ligand-efficient binding sites for fragment-sized ligands. With a challenging pathogen like $M$. tuberculosis the diverse properties of existing antimicrobials underline the need for a flexible strategy for exploring chemical space. In our experience this is a strong advantage of fragment-based design.

\section{Acknowledgments}

This work was funded by Bill \& Melinda Gates Foundation [subcontract by the Foundation for the National Institutes of Health (NIH)] (OPP1024021). T.L.B. received funding from The Wellcome Trust for facilities and support.

\section{Figure legends}


Figure 1. The fragment-based approach with methodology currently used in our laboratory. Initially, hits are identified by high-throughput biophysical or biochemical approaches, after which they are characterized structurally, thermodynamically and kinetically. Fragments are then elaborated using chemoinformatics and experimental methods.

Figure 2. X-ray crystal structures of Mycobacterium tuberculosis PanC, complexed with fragment hits and elaborated compounds. Examples of two fragments binding to different subsites of the active site are shown (a,b) (PDB codes: 3IME and 3IMC, respectively), together with hotspot maps superposed with the fragments in $\mathbf{c}$ and $\mathbf{d}$. Calculated hydrogen donor regions are colored in blue, hydrogen acceptor regions in red and apolar regions in yellow. An X-ray crystal structure of an intermediate compound is shown (e) (PDB code 3ISJ). X-ray crystal structures of compounds resulting from fragment linking and growing strategies are shown in $\mathbf{f}$ and $\mathbf{g}$ (PDB codes: 3IVX and 3IUE, respectively) together with an optimized compound (h) (PDB code 4MUK) [28,29]. Chemical structures are shown for all compounds.

Figure 3. Chemical structures of fragment hits (a,e), chemical elaboration strategy (b,f) and elaborated compounds (c,d,g,h) for Mycobacterium tuberculosis Ag85C and KasA, respectively. Two X-ray crystal structures of $M$. tuberculosis InhA ternary complexes (InhA:NAD:fragment) with fragments occupying different areas of the substrate site are shown in $\mathbf{i}$ and $\mathbf{j}$. Tyrosine 158 and phenylalanine 149 are shown in different conformations in the two structures. EthR X-ray crystal structures with fragment hits are shown in $\mathbf{k}$ and $\mathbf{m}$ (PDB codes 5F1J and 5F27, respectively). The superposition of two EthR fragment hits used for one of the merging strategies described by Nikiforov et al. [47] (I) and the resulting X-ray crystal structure of the elaborated compound (n) (PDB code 5F0F) are shown. 
Figure 4. Thymidylate kinase (TMK) and cytochrome P450 (CYP)121 X-ray crystal structures in binary complexes with fragment hits and elaborated compounds. (a,b) A TMK fragment hit and the elaboration strategy. Two different compound series (c,d and e,f) are shown with the respective X-ray crystal structures when available (PDB codes 4UNN, 4UNR, 4UNQ, respectively). (g,h) Crystal structures with a CYP121 fragment hit and elaborated compound resulting from a fragment merging followed by growing strategy described by Hudson et al. (PDB codes 4G47 and 4KTL, respectively) [61,62]. The blue, green and orange boxes (h) highlight the aromatic rings that were targeted for different fragmentation strategies [60]. (i) A crystal structure of a compound resulting from retro-fragmentation and rebuilding of compound in $\mathbf{h}$, whereas $\mathbf{j}$ depicts a crystal structure of an elaboration of compound in $\mathbf{i}$ (PDB codes $4 \mathrm{KTF}$ and 5iBE, respectively) $[60,61]$. A heme group is present in all structures. Chemical structures are shown for all compounds.

\section{References}

1 Wallis, R.S. et al. (2016) Tuberculosis--advances in development of new drugs, treatment regimens, host-directed therapies, and biomarkers. Lancet Infect. Dis. 16, e34-46

2 Koul, A. et al. (2011) The challenge of new drug discovery for tuberculosis. Nature 469, 483-490

3 Payne, D.J. et al. (2007) Drugs for bad bugs: confronting the challenges of antibacterial discovery. Nat. Rev. Drug Discov. 6, 29-40

$4 \quad$ Zumla, A. et al. (2013) Advances in the development of new tuberculosis drugs and treatment regimens. Nat. Rev. Drug Discov. 12, 388-404

5 Prideaux, B. et al. (2015) The association between sterilizing activity and drug distribution into tuberculosis lesions. Nat. Med. 21, 1223-1227

$6 \quad$ Blundell, T.L. et al. (2002) High-throughput crystallography for lead discovery in drug design. Nat. Rev. Drug Discov. 1, 45-54

7 Mashalidis, E.H. et al. (2013) A three-stage biophysical screening cascade for fragmentbased drug discovery. Nat. Protoc. 8, 2309-2324

8 Erlanson, D.A. et al. (2016) Twenty years on: the impact of fragments on drug discovery. Nat. Rev. Drug Discov. 15, 605-619

9 Scott, D.E. et al. (2012) Fragment-based approaches in drug discovery and chemical biology. Biochemistry 51, 4990-5003 
Murray, C.W. and Rees, D.C. (2009) The rise of fragment-based drug discovery. Nat. Chem. 1, 187-192

11 Niesen, F.H. et al. (2007) The use of differential scanning fluorimetry to detect ligand interactions that promote protein stability. Nat. Protoc. 2, 2212-2221

12 Vivoli, M. et al. (2014) Determination of protein-ligand interactions using differential scanning fluorimetry. J. Vis. Exp. 91, 51809

13 Dalvit, C. et al. (2001) WaterLOGSY as a method for primary NMR screening: practical aspects and range of applicability. J. Biomol. NMR 21, 349-359

14 Klages, J. et al. (2007) NMR-based screening: a powerful tool in fragment-based drug discovery. Analyst 132, 693-705

15 Mayer, M. and Meyer, B. (1999) Characterization of ligand binding by saturation transfer difference NMR spectroscopy. Angew. Chem. Int. Ed. Engl. 38, 1784-1788

16 Hajduk, P.J. et al. (1997) One-dimensional relaxation- and diffusion-edited NMR methods for screening compounds that bind to macromolecules. J. Am. Chem. Soc. $119,12257-12261$

17 Navratilova, I. and Hopkins, A.L. (2010) Fragment screening by surface plasmon resonance. ACS Med. Chem. Lett. 1, 44-48

18 Wartchow, C.A. et al. (2011) Biosensor-based small molecule fragment screening with biolayer interferometry. J. Comput. Aided Mol. Des. 25, 669-676

19 Maple, H.J. et al. (2012) Automated protein-ligand interaction screening by mass spectrometry. J. Med. Chem. 55, 837-851

20 Linke, P. et al. (2016) An automated microscale thermophoresis screening approach for fragment-based lead discovery. J. Biomol. Screen. 21, 414-421

21 Schiebel, J. et al. (2015) One question, multiple answers: biochemical and biophysical screening methods retrieve deviating fragment hit lists. ChemMedChem 10, 15111521

22 Lawson, A.D. (2012) Antibody-enabled small-molecule drug discovery. Nat. Rev. Drug Discov. 11, 519-525

23 Renaud, J.P. et al. (2016) Biophysics in drug discovery: impact, challenges and opportunities. Nat. Rev. Drug Discov. 15, 679-698

24 Hall, D.R. et al. (2015) Lessons from hot spot analysis for fragment-based drug discovery. Trends Pharmacol. Sci. 36, 724-736

25 Radoux, C.J. et al. (2016) Identifying interactions that determine fragment binding at protein hotspots. J. Med. Chem. 59, 4314-4325

26 Leonardi, R. et al. (2005) Coenzyme A: back in action. Prog. Lipid Res. 44, 125-153

27 Silvestre, H.L. et al. (2013) Integrated biophysical approach to fragment screening and validation for fragment-based lead discovery. Proc. Natl. Acad. Sci. U. S. A. 110, 1298412989

28 Hung, A.W. et al. (2009) Application of fragment growing and fragment linking to the discovery of inhibitors of Mycobacterium tuberculosis pantothenate synthetase. Angew. Chem. Int. Ed. Engl. 48, 8452-8456

29 Hung, A.W. et al. (2016) Optimization of inhibitors of Mycobacterium tuberculosis pantothenate synthetase based on group efficiency analysis. ChemMedChem 11, 3842

30 Jankute, M. et al. (2015) Assembly of the mycobacterial cell wall. Annu. Rev. Microbiol. 69, 405-423

31 Puech, V. et al. (2002) Evidence for a partial redundancy of the fibronectin-binding proteins for the transfer of mycoloyl residues onto the cell wall arabinogalactan termini of Mycobacterium tuberculosis. Mol. Microbiol. 44, 1109-1122

32 Belisle, J.T. et al. (1997) Role of the major antigen of Mycobacterium tuberculosis in cell wall biogenesis. Science 276, 1420-1422 
33 Jackson, M. et al. (1999) Inactivation of the antigen 85C gene profoundly affects the mycolate content and alters the permeability of the Mycobacterium tuberculosis cell envelope. Mol. Microbiol. 31, 1573-1587

34 Favrot, L. et al. (2013) Mechanism of inhibition of Mycobacterium tuberculosis antigen 85 by ebselen. Nat. Commun. 4, 2748

35 Scheich, C. et al. (2010) Novel small molecule inhibitors of MDR Mycobacterium tuberculosis by NMR fragment screening of antigen 85C. J. Med. Chem. 53, 8362-8367

36 Bhatt, A. et al. (2005) Conditional depletion of KasA, a key enzyme of mycolic acid biosynthesis, leads to mycobacterial cell lysis. J. Bacteriol. 187, 7596-7606

37 Wang, J. et al. (2006) Platensimycin is a selective FabF inhibitor with potent antibiotic properties. Nature 441, 358-361

38 Price, A.C. et al. (2001) Inhibition of beta-ketoacyl-acyl carrier protein synthases by thiolactomycin and cerulenin. Structure and mechanism. J. Biol. Chem. 276, 6551-6559

39 Kapilashrami, K. et al. (2013) Thiolactomycin-based beta-ketoacyl-AcpM synthase A (KasA) inhibitors: fragment-based inhibitor discovery using transient one-dimensional nuclear overhauser effect NMR spectroscopy. J. Biol. Chem. 288, 6045-6052

40 Bernardes-Genisson, V. et al. (2013) Isoniazid: an update on the multiple mechanisms for a singular action. Curr. Med. Chem. 20, 4370-4385

41 Phelan, J. et al. (2016) Mycobacterium tuberculosis whole genome sequencing and protein structure modelling provides insights into anti-tuberculosis drug resistance. BMC Medicine 14, 31

42 Encinas, L. et al. (2014) Encoded library technology as a source of hits for the discovery and lead optimization of a potent and selective class of bactericidal direct inhibitors of Mycobacterium tuberculosis InhA. J. Med. Chem. 57, 1276-1288

43 Manjunatha, U.H. et al. (2015) Direct inhibitors of InhA are active against Mycobacterium tuberculosis. Sci. Transl. Med. 7, 269ra263

44 Sink, R. et al. (2015) Design, synthesis, and evaluation of new thiadiazole-based direct inhibitors of enoyl acyl carrier protein reductase $(\operatorname{InhA})$ for the treatment of tuberculosis. J. Med. Chem. 58, 613-624

45 Wang, F. et al. (2007) Mechanism of thioamide drug action against tuberculosis and leprosy. J. Exp. Med. 204, 73-78

46 Willand, N. et al. (2009) Synthetic EthR inhibitors boost antituberculous activity of ethionamide. Nat. Med. 15, 537-544

$47 \quad$ Nikiforov, P.O. et al. (2016) A fragment merging approach towards the development of small molecule inhibitors of Mycobacterium tuberculosis EthR for use as ethionamide boosters. Org. Biomol. Chem. 14, 2318-2326

48 Villemagne, B. et al. (2014) Ligand efficiency driven design of new inhibitors of Mycobacterium tuberculosis transcriptional repressor EthR using fragment growing, merging, and linking approaches. J. Med. Chem. 57, 4876-4888

49 Surade, S. et al. (2014) A structure-guided fragment-based approach for the discovery of allosteric inhibitors targeting the lipophilic binding site of transcription factor EthR. Biochem. J. 458, 387-394

50 Frenois, F. et al. (2004) Structure of EthR in a ligand bound conformation reveals therapeutic perspectives against tuberculosis. Mol. Cell 16, 301-307

51 Kumar, A. et al. (2010) New molecular scaffolds for the design of Mycobacterium tuberculosis type II dehydroquinase inhibitors identified using ligand and receptor based virtual screening. J. Mol. Model. 16, 693-712

52 Tran, A.T. et al. (2012) Elucidation of Mycobacterium tuberculosis type II dehydroquinase inhibitors using a fragment elaboration strategy. ChemMedChem 7, 1031-1043 
53 Van Calenbergh, S. et al. (2012) Drug design and identification of potent leads against mycobacterium tuberculosis thymidine monophosphate kinase. Curr. Top. Med. Chem. $12,694-705$

$54 \quad$ Naik, M. et al. (2015) Structure guided lead generation for M. tuberculosis thymidylate kinase (Mtb TMK): discovery of 3-cyanopyridone and 1,6-naphthyridin-2-one as potent inhibitors. J. Med. Chem. 58, 753-766

55 Cole, S.T. et al. (1998) Deciphering the biology of Mycobacterium tuberculosis from the complete genome sequence. Nature 393, 537-544

56 McLean, K.J. et al. (2006) The preponderance of P450s in the Mycobacterium tuberculosis genome. Trends Microbiol. 14, 220-228

57 McLean, K.J. et al. (2008) Characterization of active site structure in CYP121. A cytochrome P450 essential for viability of Mycobacterium tuberculosis H37Rv. J. Biol. Chem. 283, 33406-33416

58 Seward, H.E. et al. (2006) Crystal structure of the Mycobacterium tuberculosis P450 CYP121-fluconazole complex reveals new azole drug-P450 binding mode. J. Biol. Chem. 281, 39437-39443

59 McLean, K.J. et al. (2002) Azole antifungals are potent inhibitors of cytochrome P450 mono-oxygenases and bacterial growth in mycobacteria and streptomycetes. Microbiology 148, 2937-2949

60 Kavanagh, M.E. et al. (2016) Fragment-based approaches to the development of Mycobacterium tuberculosis CYP121 inhibitors. J. Med. Chem. 59, 3272-3302

61 Hudson, S.A. et al. (2013) Overcoming the limitations of fragment merging: rescuing a strained merged fragment series targeting Mycobacterium tuberculosis CYP121. ChemMedChem 8, 1451-1456

62 Hudson, S.A. et al. (2012) Application of fragment screening and merging to the discovery of inhibitors of the Mycobacterium tuberculosis cytochrome P450 CYP121. Angew. Chem. Int. Ed. Engl. 51, 9311-9316

63 Davis, H.J. et al. (2016) Spirooxindoles as novel 3D-fragment scaffolds: synthesis and screening against CYP121 from M. tuberculosis. Bioorg. Med. Chem. Lett. 26, 37353740

64 Danilchanka, O. et al. (2008) Role of porins for uptake of antibiotics by Mycobacterium smegmatis. Antimicrob. Agents Chemother. 52, 3127-3134

65 Gopal, P. and Dick, T. (2014) Reactive dirty fragments: implications for tuberculosis drug discovery. Curr. Opin. Microbiol. 21, 7-12 


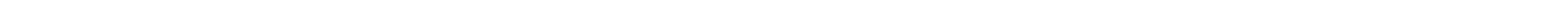




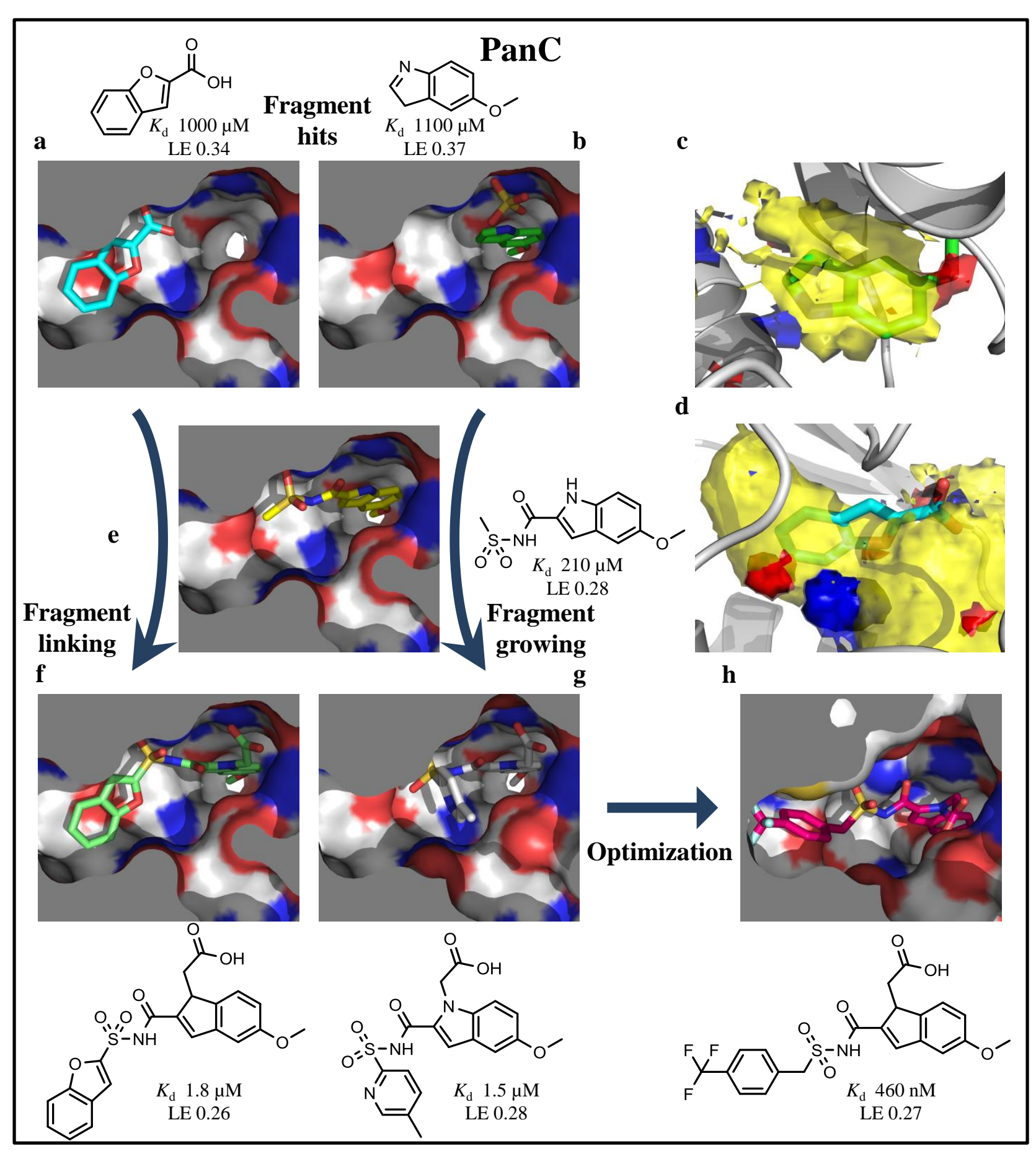




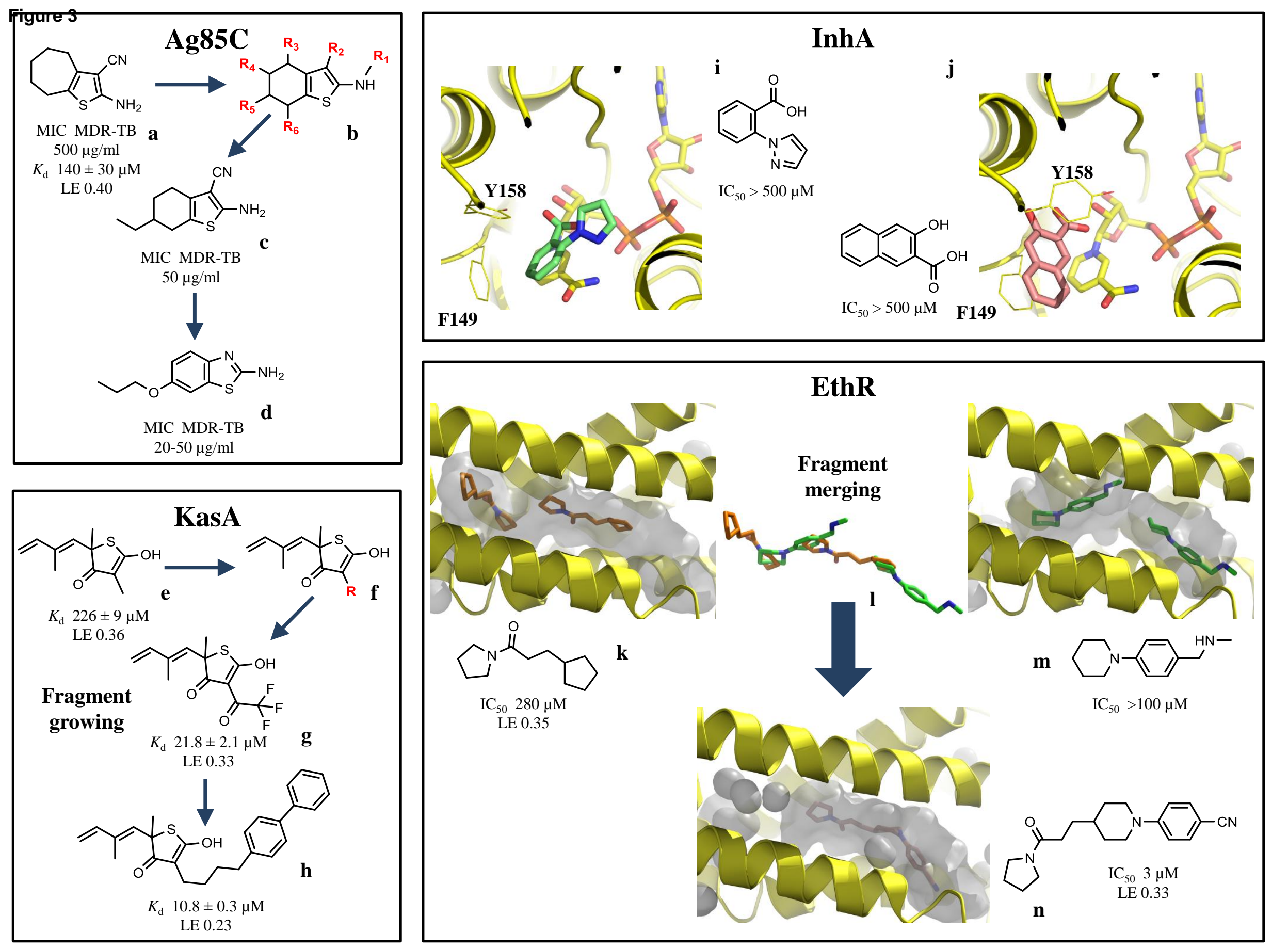




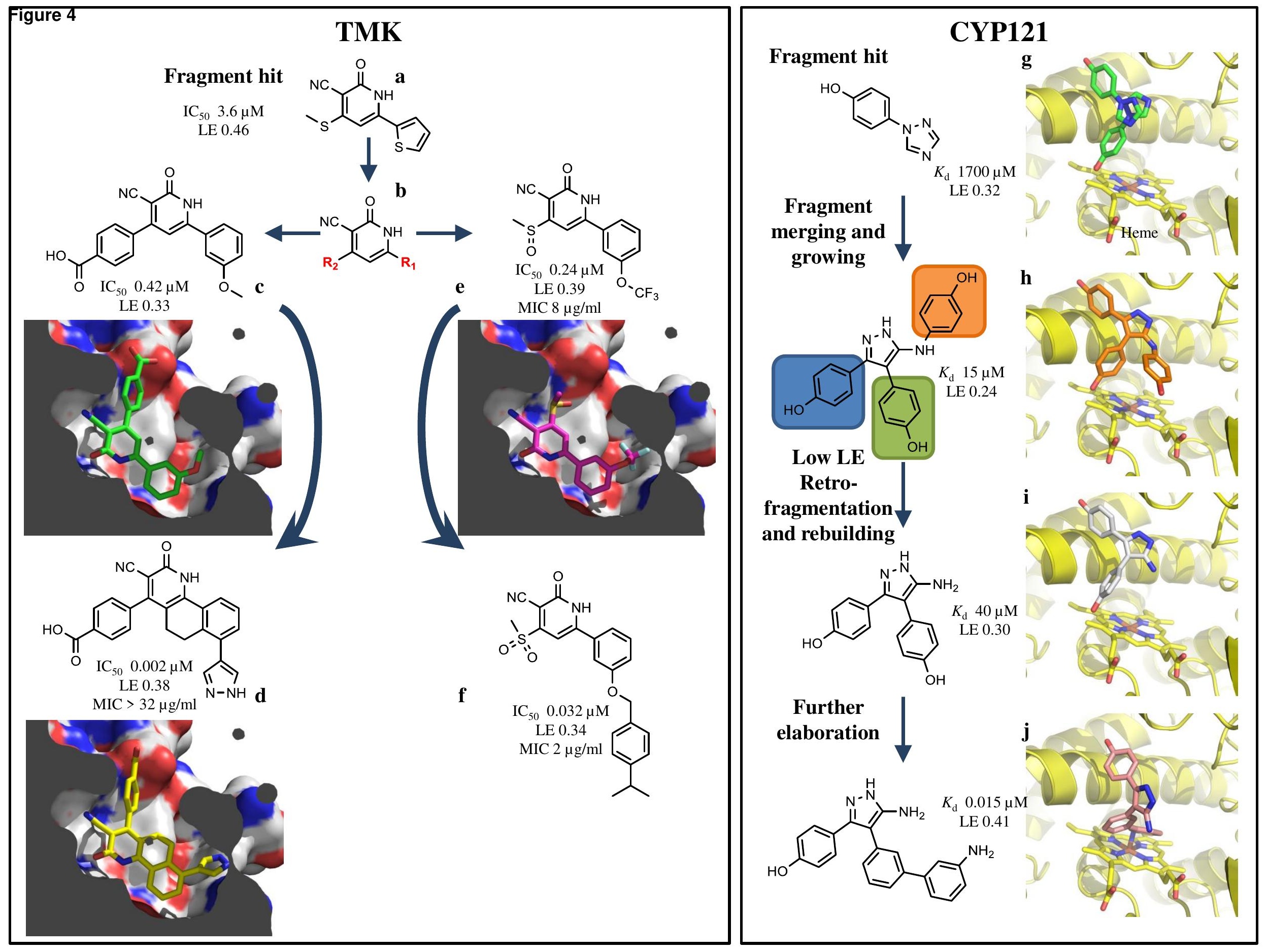

